\title{
Newton's Cradle and Quantum Observation
}

\author{
Terry B. Bollinger (1) 1 \\ ${ }^{1}$ Apabistia Research, Ashburn, VA, USA 20147
}

(Incepted 27 October 2019; Published 27 October 2019; Tweaked 2021-07-28.20:37)*

\begin{abstract}
This paper defines quantum observation as conversion of tiny (attojoule or less) packets of energy and momentum into statistically irreversible Boltzmann heat. Under this definition, quantum waves never "collapse" but instead grow so fractally convoluted in one region of space that their entangled properties in remote regions of space become statistically irreversible and persistent over time. The implication is that far from being a rare phenomenon that depends on such hard-to-define phenomena as human awareness, quantum observation via atto-scale creation of Boltzmann heat is the most common of all quantum phenomena and the underlying basis for the very existence of "classical" matter and objects.
\end{abstract}

DOI: https://doi.org/10.48034/20191027

\section{NEWTON'S CRADLE AS A WAVE MODEL}

Most folks have seen a Newton's Cradle. It is a chain of barely touching steel balls, each held by two strings to keep their motions aligned.

If you drop a ball at one end of a Cradle, its impact transmits a momentum impulse to the other end of the chain, launching the end ball into motion. The end ball then reverses direction and begins the process over:

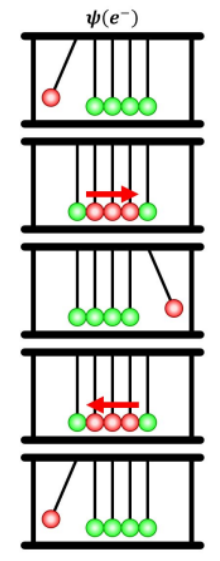

FIG. 1. Electron wave functions as an adiabatic oscillations.

Published by Apabistia Press under the Creative Commons Attribution 4.0 International License. You are free to redistribute this article as you choose, provided only that you preserve the author attribution, article title, journal citation, and DOI.

${ }^{*}$ This document uses Kenobi versioning. The online name always points to the most recent document version, while obl, $o b 2, \ldots$ suffixes indicate obsolete releases of the document.
This is about: Quantum thermodynamics,

Quantum nonlocality, Quantum foundations
The Cradle is a great demo of momentum transfer, but it is also an excellent example of a time-reversible oscillatory process, since a video of a Cradle in action looks the same whether it is played forward or backward.

\section{THE IMPACT OF THERMAL COMPLEXITY}

Now, let's change things up a bit by replacing the final ball with a cluster of much smaller balls $\beta$, each suspended from one string instead of two. What happens?

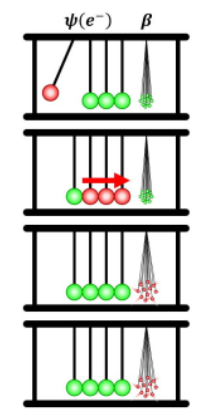

FIG. 2. Electron observation as nonadiabic heat generation.

The momentum is transferred as before into the final unit, but then everything falls apart, literally. When the momentum impulse hits, the less constrained tiny balls scatter like billiard balls after a hard cue ball break. The original sharp momentum pulse is blurred and diffused over time, making its reconstruction extremely unlikely. Time symmetry is lost, since the process can no longer repeat itself.

Another way to describe this loss of time symmetry is to say the Cradle is 'observed' by the chaotic transformation 
that takes place within its more complex end unit. It's an observation in the sense that the end unit keeps an energy 'record' of the initial ball impact, the one that took place at the other end of the chain. Furthermore, since timereversibility has been lost, that now-singular impact takes on a temporal uniqueness that makes it more like a classical event.

\section{PHOTONS AS ADIABATIC OSCILLATIONS}

Much like the chain of balls in Newton's Cradle, a photon also carries momentum across space. And also like Newton's Cradle, a photon remains 'simple' and timereversible only if both ends of its momentum transmission path remain simple and quantum. While that sounds unlikely, it actually happens all the time. We call it 'transparency', which is a rather amazing bit of physics in its own right.

Now, let's take another look at the two-hole electron diffraction experiment.

A photon that bounces off of an electron does not immediately become 'classical', since both the electron and photon are simple and thus capable of time-reversal. However, if the photon next hits a bit of thermal matter that not only absorbs but shreds and purees its momentum across a large ensemble of jiggling atoms, its time-reversal become statistically unlikely, and thus the photon becomes part of classical physics. Notice, however - and this is important - that this simplicity is lost at both ends of the photon chain, not just at the absorption or detection end. The original electron emission event also loses its ability to be oscillatory, which means that it too must become classical.

This situation of an initially reversible electron-photon interaction becoming statistically irreversible through chaotic shredding of the (possibly very remote) photon end of a momentum pair can also be modeled by with Newton's Cradle. This time two end-to-end clusters are used to represent a reversible momentum pair:

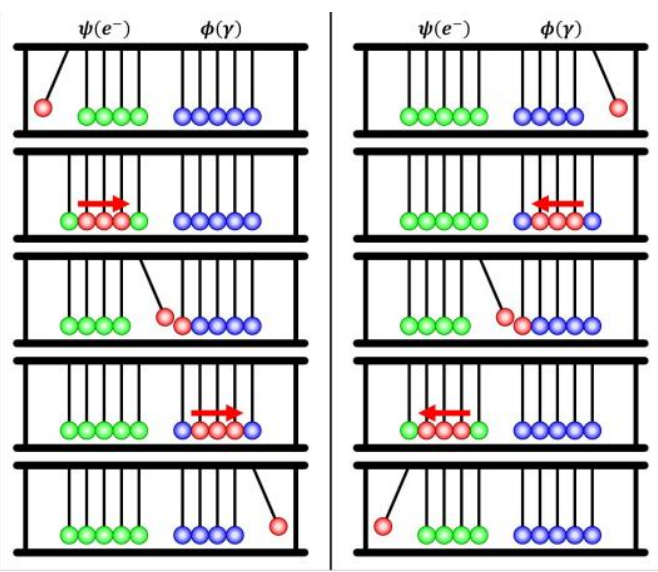

FIG. 3. Reversible adiabatic photon observation of an electron.
The left-hand cradle cluster represents electron momentum, while the right-hand cluster represents photon momentum.

\section{NONADIABATIC PHOTON “OBSERVERS"}

The photon end of the momentum pair can be "shredded" (made statistically irreversible) if it encounters an adiabatic rhodopsin molecule in a human eye, whose pico-heating locks in irreversible Boltzmann $(\beta)$ time:

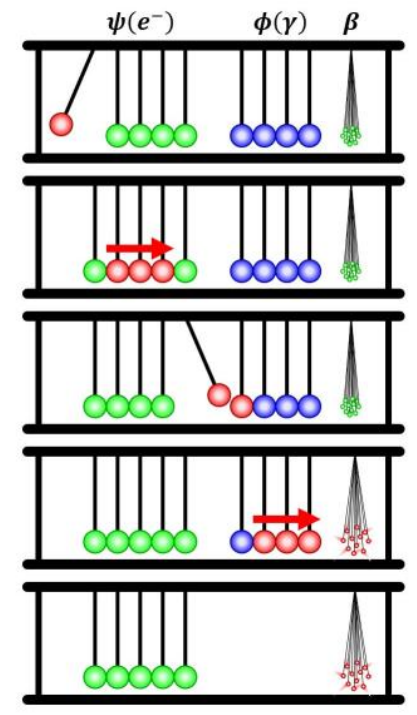

FIG. 4. Irreversible nonadiabatic photon electron observation.

Even if this photon shredding occurs at a distant location, what was potentially a time-reversible situation becomes insurmountably unlikely to reverse after the shredding event. This statistically insurmountable break in time symmetry effectively localizes the left-side wave function of the electron, forcing the electron to move forward in history with fewer options. The electron has been observed.

Stated a bit differently: Chaotic absorption of a photon forces its entire momentum history to become classical. Thus the absorption also makes the entangled emitting electron classical, since the simple oscillatory (quantum) behavior of that electron is disrupted just as much as that of the photon. It is as if the observer 'donates' a bit of their own classical reality to the electron via momentum entanglement, no matter how distant the electron may be.

Decoherence has it mostly right in asserting that interactions with larger objects degrade quantum behavior. However, the deeper message is that there is a statistical continuum in all forms of quantum observation. Even a dust speck can 'observe' quantum events, provided only that it is complex enough to provide statistically irreversible shredding of one end of a previously simple wave function. 


\section{OBSERVATION AS CREATING HEAT}

The bottom line is this: For matters quantum, it's not the consciousness of the observer that matters. It's the thermal chaos in the rhodopsin of her eyes. And yes, there probably should be a song about that... :)

\section{ACKNOWLEDGEMENTS}

I want to thank Sabine Hossenfelder for posting the essay that prompted this analysis [1], and posting the first text version of this article as a comment on her essay [2].

\section{APPENDIX A: USE OF INFORMALITY TO CONVEY NEW CONCEPTS}

Human cognition is a curious and profoundly complex phenomenon. When I first posted the text of this paper on the Backreaction site, my intent was not to quantify the ideas precisely but to introduce a new perspective on topics that are mysterious indeed when analyzed from some set of axioms that are deeply ingrained in how we perceive and interpret the world that it does not usually occur to us to question them. The axiom in question, in this case, is the idea that classical matter and objects are the best starting point for comprehending and analyzing reality, despite our full intellectual awareness over the last century (since the 1920s) that every classical object is at its finest levels composed of quantum wave functions.

If instead, concepts such as location and persistence over time are emergent phenomena that grow out of those wave functions through the full scope of how they interact, a different picture emerges. For example, we know that the wave function of an atom in deep space must expand and fill that space over time. Yet, at the same time, we know that no such expansion occurs when the same atom resides within thermal matter. A surprisingly simple resolution of these seemingly contradictory views, which this paper explores, is that the atom within thermal matter is observed every time it bumps into other atoms. Far from collapsing, the complete topology of the wave function thus becomes unimaginably more complex over time. As a result, the localization within the crystal of the mass, spin, and charge components of the atom's wave function become a matter of quantum compromise. This trade-off reduces overall energy at the cost of continually and irreversibly increasing the complexity of the momentum components of that same wave function.

Newton's Cradle provides a helpful visual image for all of this since as long as a clock of any sort repeats its cycles without disruption, it has the same timeless properties as a quantum wave function. The difference is that actual clocks are embedded in time and always have a real state, whereas quantum wave function "clocks" are truly timeless and acquire classical state only when inspected.
[1] S. Hossenfelder, "What is the quantum measurement problem?” Backreaction, October 22, 2019. https://backreaction.blogspot.com/2019/10/ what-is-quantum-measurement-problem.html

[2] T. Bollinger, "Newton's Cradle and Quantum Observation.” Backreaction, October 27, 2019. https://backreaction.blogspot.com/2019/10/ what-is-quantum-measurement-problem.html ?showComment $=$ $\underline{1572213322428 \# \text { c6028159424345209571 }}$ 